\title{
Treadmill exercise improves motor and memory functions in cerebral palsy rats through activation of PI3K-Akt pathway
}

\author{
Sun-Young Jung', Dae-Young Kim²,* \\ 'Department of Physical Therapy, Hosan University, Gyeongsan, Korea \\ ${ }^{2}$ Department of Sports Healthcare, College of Humanities \& Social Sciences, Inje University, Gimhae, Korea
}

Cerebral palsy (CP) is a chronic disorder characterized by physical disability and disruption of brain function. We evaluated the effects of treadmill exercise on motor and memory functions in relation with phosphatidylinositol 3-kinase (PI3K)-Akt pathway using CP rat model. Rota-rod test, step-down avoidance task, 5-bromo-2'-deoxyuridine (BrdU) immunohistochemistry, and western blot for synapsin I, postsynaptic density-95 (PSD-95), PI3K, Akt, and glycogen synthase kinase-3 $\beta$ (GSK-3 3 ) were performed. CP was induced by maternal lipopolysaccharide (LPS)-injection with sensorimotor restriction. Five weeks after birth, the rats in the exercise groups were made to run on the treadmill for 30 min per one day, 5 times a week, during 4 weeks. Motor and memory functions were impaired in the LPS-induced CP rats and treadmill exercise increased motor and memory functions in the CP rats. Cell proliferation in the hippocampus was suppressed in the LPS-induced $\mathrm{CP}$ rats and treadmill exercise increased hippocampal cell proliferation in the CP rats. Expressions of synapsin I, PSD-95, phosphorylated (p)$\mathrm{PI} 3 \mathrm{~K}$, and $\mathrm{p}$-Akt were decreased in the LPS-induced CP rats and treadmill exercise enhanced the expressions of synapsin I, PSD-95, p-PI3K, and $p$-Akt in the CP rats. GSK-3 $\beta$ expression was increased in the LPS-induced CP rats and treadmill exercise suppressed GSK-3 $\beta$ expression in the CP rats. The present results suggest that treadmill exercise might improve motor and memory functions through activation of PI3K-Akt pathway.

Keywords: Cerebral palsy, Treadmill exercise, Motor function, Memory function, PI3K-Akt pathway

\section{INTRODUCTION}

Cerebral palsy $(\mathrm{CP})$ is a chronic disorder characterized by physical disability and disruption of brain function, resulting in inadequate control of movement and cognition (Derrick et al., 2004). In the $\mathrm{CP}$ patients, secondary motor impairments, such as abnormal motor patterns and inadequate postures, persist throughout the lifespan and disturb normal development of children (Damiano et al., 2009).

Various studies using CP animal models have attempted to evaluate the pathophysiology of CP. However, CP animal models do not present the characteristic motor disorders observed in $\mathrm{CP}$ patients. In order to present similar motor disorders observed in CP patients, Strata et al. (2004) designed a rat model for CP based on perinatal anoxia with sensorimotor restriction. Sensorimotor restriction presented decreased movement and abnormal proprioception during early stage of development, resembling abnormal motor patterns observed in CP patients (Strata et al., 2004). Sensorimotor restriction with anoxia produced long-lasting deficits, such as reduced body growth rate, increased muscle tone, and disorganization of primary motor and somatosensory cortex (Coq et al., 2008; Marcuzzo et al., 2010). One limitation of these models is that these models did not consider the possibility of maternal infection, one of the important etiologic factors inducing CP. In this study, we made a CP animal model using lipopolysaccharide (LPS) for maternal infection with sensorimotor restriction (Stigger et al., 2011). LPS is a structural component of most gram-negative bacteria, and LPS induces cytotoxicity in the fetal brain

\footnotetext{
${ }^{*}$ Corresponding author: Dae-Young Kim (D) http://orcid.org/0000-0002-4662-4463 Department of Sports Health Care, College of Humanities \& Social Sciences, Inje University, 197 Inje-ro, Gimhae 50834, Korea

Tel: +82- 55-320-3171, Fax: +82-55-320-3545, E-mail: cdps21@inje.ac.kr Received: March 2, 2017 / Accepted: April 17, 2017
}

This is an Open Access article distributed under the terms of the Creative Commons Attribution Non-Commercial License (http://creativecommons.org/licenses/by-nc/4.0/) which permits unrestricted non-commercial use, distribution, and reproduction in any medium, provided the original work is properly cited. 
during pregnancy (Gilstrap and Ramin, 2000).

Exercise increases expression of neurotrophic factors and enhances neurogenesis (Jung et al., 2014; van Praag et al., 2005; Ying et al., 2008). Exercise activates phosphatidylinositol 3-kinase (PI3K)Akt pathway, which affects cell proliferation and migration during neurogenesis (Zhang et al., 2013; Tiwari et al., 2015). Akt is a well-characterized serine/threonine kinase and a down-stream target of PI3K that controls metabolism, cell survival, and neurogenesis (Wu et al., 2009). PI3K-Akt pathway in the brain modulates synaptic plasticity, neural survival, and cognitive function (Gaspa-

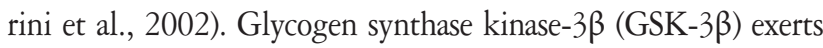
negative effect on hippocampal neurogenesis. GSK- $3 \beta$ is a downstream target of Akt and activated Akt inhibits GSK- $3 \beta$ by inducing its phosphorylation (Wu et al., 2009). Exercise activates Akt and then GSK-3 $\beta$ activity is suppressed (Sakamoto et al., 2003).

In present study, we evaluated the effects of treadmill exercise on motor and memory function focusing on neurogenesis and synaptic plasticity in relation with $\mathrm{PI} 3 \mathrm{~K}$-Akt pathway using $\mathrm{CP}$ rat model.

\section{MARERIALS AND METHODS}

\section{Animals and treatments}

Female Sprague-Dawley rats $(180 \pm 10$ g, 8 weeks old, $\mathrm{n}=20)$ were allowed to mate with male rats $(200 \pm 10 \mathrm{~g}, 10$ weeks old, $\mathrm{n}=10$ ) for $24 \mathrm{hr}$. Then, the female rats were individually housed in plastic home cages under controlled temperature $\left(20^{\circ} \mathrm{C} \pm 2^{\circ} \mathrm{C}\right)$ and a light-dark cycle consisting of $12 \mathrm{hr}$ of light and $12 \mathrm{hr}$ of darkness (lights on from 07:00 a.m. to 19:00 p.m.). Food and water were made available ad libitum.

The pregnant rats were divided into 2 groups: the control group and the LPS-injection group ( $n=4$ in each group). On the 15 th, 17 th, and 20th day of pregnancy, the pregnant rats in the LPS-injection group received $1-\mathrm{mL}$ intracervical injection of $0.15-\mathrm{mg} / \mathrm{kg}$ LPS (from Escherichia coli, serotype 055:B5, Sigma Chemical Co., St. Louis, MO, USA) suspended in pyrogen-free saline (PFS), and the pregnant rats in the control group were treated with PFS.

After birth, the neonatal rats were divided into 5 groups ( $n=8$ in each group): the control group, the $\mathrm{CP}$ group, the $\mathrm{CP}$ and exercise group, the $\mathrm{CP}$ and sensorimotor restriction group, and the $\mathrm{CP}$, sensorimotor restriction, and exercise group. The rats in the sensorimotor restriction groups were restrained for $16 \mathrm{hr}$ once a day from postnatal day 2 to postnatal day 28. The rats' hind limbs bound together with paper tape and placed in an extended position, according to the previous described method (Strata et al., 2004).

\section{Treadmill exercise protocol}

Five weeks after birth, the rats in the exercise groups were forced to run on treadmill for 30 min once a day, 5 times a week during 4 weeks. The exercise load consisted of running at a speed of $3 \mathrm{~m} / \mathrm{min}$ for the first $5 \mathrm{~min}$, at a speed of $5 \mathrm{~m} / \mathrm{min}$ for the next $5 \mathrm{~min}$, and then at a speed of $8 \mathrm{~m} / \mathrm{min}$ for the last $20 \mathrm{~min}$ with $0 \%$ grade of inclination.

\section{Rota-rod test}

In order to evaluate the motor function of the rats, the latency of the rota-rod test was conducted, as a previously described method (Marques et al., 2014). The animals were placed in a rota-rod with 60-mm diameter rod, $75 \mathrm{~mm}$ in length, rotating speed of $25 \mathrm{rpm}$. Each animal was tested 5 times with the 2-min interval between each trial, and the maximum duration of the test was $3 \mathrm{~min}$. The time spent on the rota-rod of each rat was considered as the latency to fall.

\section{Step-down avoidance task}

In order to evaluate the memory function of the rats, the latency of the step-down avoidance task was conducted, as the previously described method (Shin et al., 2013). The rats were trained in a step-down avoidance task in which they were positioned on a $7 \times 25-\mathrm{cm}$ platform with a height of $2.5 \mathrm{~cm}$, and then allowed to rest on the platform for $1 \mathrm{~min}$. The platform faced a $42 \times 25-\mathrm{cm}$ grid of parallel $0.1 \mathrm{~cm}$-caliber stainless steel bars, which were spaced $1 \mathrm{~cm}$ apart. In the training session, the animals received a 0.5-mA scramble foot shock for $2 \mathrm{sec}$ immediately upon stepping of off the platform. Retention time was assessed at $48 \mathrm{hr}$ after training session. The interval of rats stepping down and placing all 4 paws on the grid was defined as the latency of the step-down avoidance task. Any latency over $300 \mathrm{sec}$ was counted as $300 \mathrm{sec}$.

\section{Tissue preparation}

To begin the sacrificial process, the animals were fully anesthetized using Zoletil 50 (10 mg/kg intraperitoneally; Vibac Laboratories, Carros, France). After a complete lack of response was observed, the rats were transcardially perfused with 50-mM phosphate-buffered saline (PBS) and subsequently fixed with freshly prepared 500$\mathrm{mM}$ phosphate buffer (PB; $\mathrm{pH}, 7.4)$ containing $4 \%$ paraformaldehyde. The brains of the rats were removed and fixed in the same fixative overnight and then transferred into a $30 \%$ sucrose solution for cryoprotection. Serial coronal sections of $20-\mu \mathrm{m}$ thickness were obtained using a freezing microtome (Leica, Nussloch, Germany). 


\section{Immunohistochemistry for 5-bromo-2'-deoxyuridine}

For the detection of newly generated cells in the dentate gyrus, immunohistochemistry for 5-bromo-2'-deoxyuridine (BrdU) immunohistochemistry was performed, according to a previously described method (Shin et al., 2013). The brain sections were first permeabilized by incubation in $0.5 \%$ Trioton X-100 in PBS for $20 \mathrm{~min}$, then pretreated in $50 \%$ formamide $-2 \mathrm{x}$ standard saline citrate at $65^{\circ} \mathrm{C}$ for $2 \mathrm{hr}$, denaturated in $2 \mathrm{~N} \mathrm{HCl}$ at $37^{\circ} \mathrm{C}$ for 30 min, and rinsed twice in $100-\mathrm{mM}$ sodium borate $(\mathrm{pH}, 8.5)$. Thereafter, the sections were incubated overnight at $4^{\circ} \mathrm{C}$ with mouse monoclonal anti-BrdU antibody (1:600; Roche, Mannheim, Germany). Then, the sections were washed three times with PBS and incubated for $90 \mathrm{~min}$ with the biotionylated mouse secondary antibody (1:200; Vector Laboratories, Burlingame, CA, USA). Then, the sections were incubated with avidin-peroxidase complex (1:100; Vector Laboratories). For visualization, the section were incubated in $50-\mathrm{mM}$ Tris- $\mathrm{HCl}(\mathrm{pH}, 7.6)$ containing $0.02 \% 3,3^{\prime}$-diaminobenzidine (DAB), $40-\mathrm{mg} / \mathrm{mL}$ nickel chloride and $0.03 \%$ hydrogen peroxide for $5 \mathrm{~min}$.

After BrdU-specific staining, counter-staining was performed on the same sections using a mouse monoclonal antineuronal nucleic antibody (1:300; Chemicon International, Temecula, CA, USA). The sections were washed three times with PBS and incubate for 1 hr with a biotinylated anti-mouse secondary antibody. For staining, the sections were incubated in a reaction mixture consisting of $0.02 \% \mathrm{DAB}$ and $0.03 \%$ hydrogen peroxide for $5 \mathrm{~min}$. The sections were finally mounted onto gelatin-coated slides. The slides were air dried overnight at room, and coverslips were mounted with Permount (Thermo Fisher Scientific Inc., Waltham, MA, USA).

The numbers of BrdU-positive cells in the dentate gyrus was counted hemilaterally under a light microscope (Olympus, Tokyo, Japan), and they were expressed as the number of cells per square millimeter in the dentate gyrus. The area of the dentate gyrus was measured with the Image-Pro Plus image analysis system (Media Cyberbetics Inc., Silver Sprng, MD, USA).

\section{Western blotting}

Western blot was performed, according to a previously described method (Kim et al., 2015). Protein extracts from hippocampal tissues were separated by sodium dodecyl sulfate-polyacrylamide gel electrophoresis. Protein separation was performed using $10 \%$ polyacrylamide with $0.05 \%$ bis-acrylamide. Proteins were then transferred to nitrocellulose and the blots were probed with anti-PI3K mouse monoclonal antibody (1:1,000, Santa Cruz Biotechnology, Santa Cruz, CA, USA), anti-Akt rabbit polyclonal antibody (1:1,000, Cell Signaling Technology Inc., Beverly, MA, USA), anti-GSK-3 $\beta$ rabbit polyclonal antibody (1:1,000, Santa Cruz Biotechnology), anti-synapsin I mouse monoclonal antibody (1:1,000, Santa Cruz Biotechnology), and anti-postsynaptic density-95 (PSD-95) mouse monoclonal antibody (1:1,000, Santa Cruz Biotechnology). Peroxidase anti-rabbit IgG (1:5,000, Vector Laboratories), and peroxidase anti-mouse $\operatorname{IgG}(1: 10,000$, Vector Laboratories) were used as the secondary antibodies. Immunoreactivity was detected by enhanced chemiluminescence (ECL) detection kit (Santa Cruz Biotechnology).

\section{Data analysis}

Differences among the groups were evaluated using IBM SPSS Statistics ver. 23.0 (IBM Co., Armonk, NY, USA) by the one-way analysis of variance followed by Duncan post hoc test. All values are expressed as the mean \pm standard error of the mean. Statistically significant differences were established at $P<0.05$.

\section{RESULTS}

\section{Motor function}

Motor function (balance and coordination) was measured using the rota-rod test (Fig. 1). The latency in the $\mathrm{CP}$ rats were shorter than that in the control group $(P<0.05)$. In contrast, treadmill exercise increased the latency in the $\mathrm{CP}$ rats $(P<0.05)$.

\section{Memory function}

Short-term memory was measured using the step-down avoid-

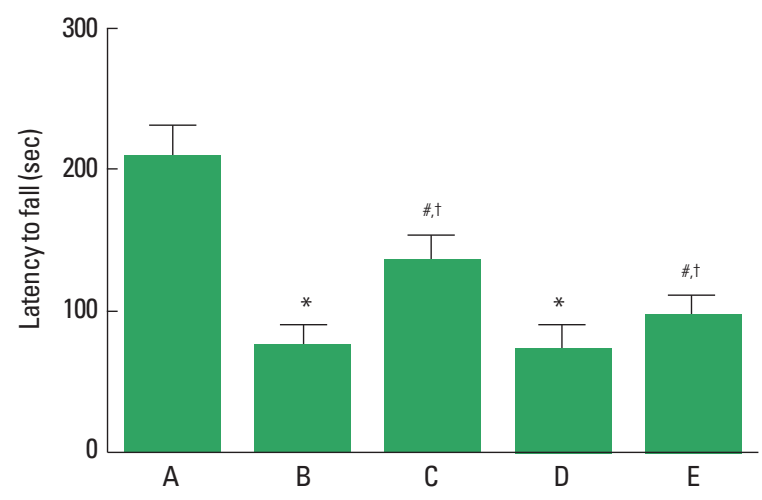

Fig. 1. Effects of treadmill exercise on motor function. A, control group; B, cerebral palsy group; $C$, cerebral palsy and exercise group; $D$, cerebral palsy and sensorimotor restriction group; $\mathrm{E}$, cerebral palsy, sensorimotor restriction, and exercise group. The data are presented as the mean \pm standard error of the mean. ${ }^{*} P<0.05$ compared to the control group. ${ }^{\#} P<0.05$ compared to the cerebral palsy group. ${ }^{\dagger} P<0.05$ compared to the cerebral palsy and sensorimotor restriction group. 


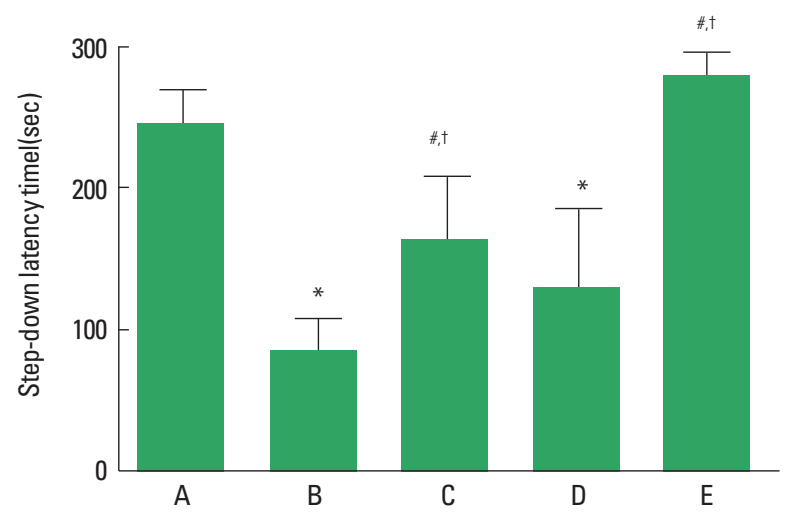

Fig. 2. Effects of treadmill exercise on memory function. $A$, control group; $B$, cerebral palsy group; $\mathrm{C}$, cerebral palsy and exercise group; $\mathrm{D}$, cerebral palsy and sensorimotor restriction group; $\mathrm{E}$, cerebral palsy, sensorimotor restriction, and exercise group. The data are presented as the mean \pm standard error of the mean. ${ }^{*} P<0.05$ compared to the control group. ${ }^{\sharp} P<0.05$ compared to the cerebral palsy group. ${ }^{\dagger} P<0.05$ compared to the cerebral palsy and sensorimotor restriction group.

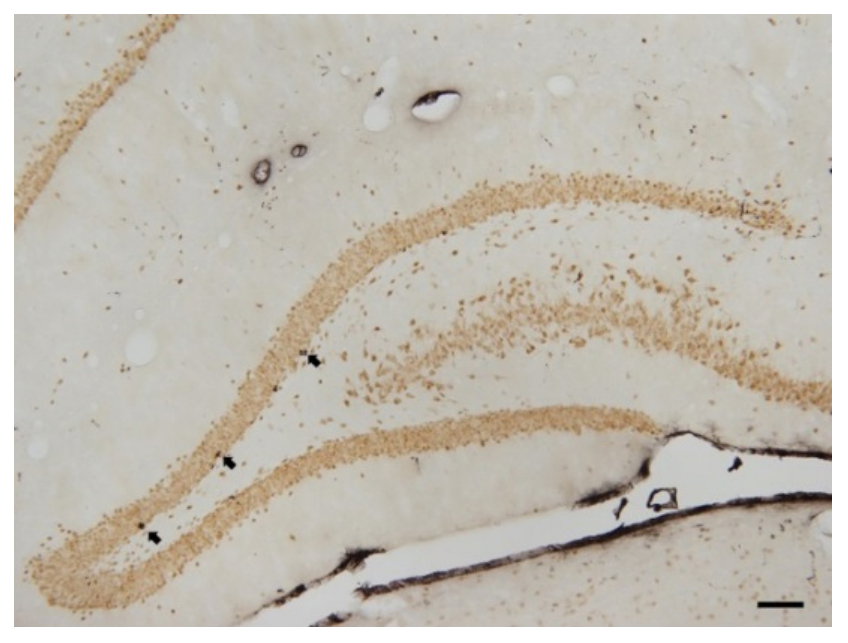

Fig. 3. Photomicrographs of 5-bromo-2'-deoxyuridine (BrdU)-positive cells in the hippocampal dentate gyrus of the control group. The scale bar represents $200 \mu \mathrm{m}$. Arrows indicate BrdU-positive cells.

ance task (Fig. 2). The latency in the $\mathrm{CP}$ rats was lower than that in the control group $(P<0.05)$. In contrast, treadmill exercise increased the latency in the $\mathrm{CP}$ rats $(P<0.05)$.

\section{Cell proliferations in the hippocampal dentate gyrus}

Photomicrograph of BrdU-positive cells in the hippocampal dentate gyrus is shown in Fig. 3. Cell proliferation in the hippocampal dentate gyrus was decreased in the $\mathrm{CP}$ rats $(P<0.05)$, in contrast, treadmill exercise enhanced cell proliferation in the $\mathrm{CP}$ rats $(P<0.05)$ (Fig. 4).

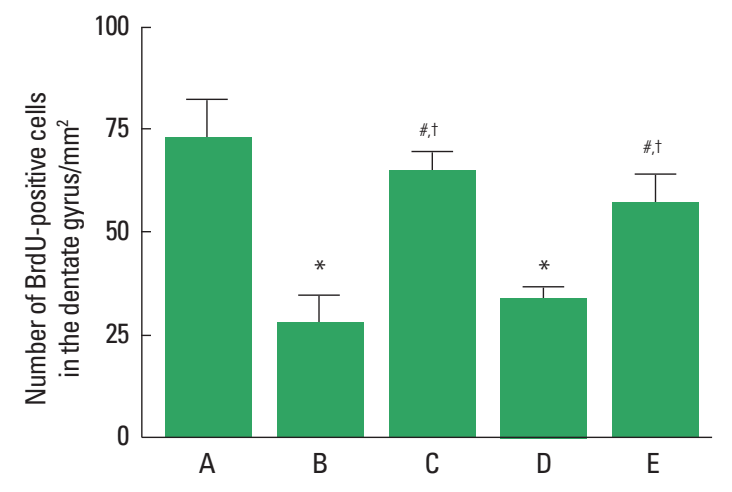

Fig. 4. Effect of treadmill exercise on the number of 5-bromo-2'-deoxyuridine (BrdU)-positive cells in the hippocampal dentate gyrus. A, control group; B, cerebral palsy group; $\mathrm{C}$, cerebral palsy and exercise group; $\mathrm{D}$, cerebral palsy and sensorimotor restriction group; $\mathrm{E}$, cerebral palsy, sensorimotor restriction, and exercise group. The data are presented as the mean \pm standard error of the mean. ${ }^{*} P<0.05$ compared to the control group. ${ }^{\#} P<0.05$ compared to the cerebral palsy group. ${ }^{\dagger} P<0.05$ compared to the cerebral palsy and sensorimotor restriction group.

\section{Synapsin I, PSD-95, p-PI3K, p-Akt, GSK-3ß expressions in the hippocampus}

Western blot analysis for the expressions of synapsin I, PSD-95, p-PI3K, p-Akt, GSK-3ß in the hippocampus was performed (Fig. 5). Expressions of synapsin I, PSD-95, p-PI3K, and p-Akt in the hippocampus were decreased in the $\mathrm{CP}$ rats $(P<0.05)$, in contrast, treadmill exercise increased synapsin I, PSD-95, p-PI3K, and $\mathrm{p}$-Akt expressions in the in the $\mathrm{CP}$ rats $(P<0.05)$. The expression of GSK-3 $\beta$ in the hippocampus was increased in the CP rats $(P<0.05)$, in contrast, treadmill exercise suppressed GSK-3 $\beta$ expression in the $\mathrm{CP}$ rats $(P<0.05)$.

\section{DISCUSSION}

$\mathrm{CP}$ is a group of movement and posture disorders that cause activity limitation (Bax et al., 2005). There is no definitive treatment for CP, however, improved physical conditioning decreases secondary impairments (Damiano, 2006).

In our study, sensorimotor restriction did not aggravated motor and memory impairments in the LPS-induced CP rats. Early experience-dependent movements played a crucial role in shaping normal behavioral motor abilities (Strata et al., 2004) and treadmill running improved deteriorated memory function (Kim et al., 2015). In the present study, treadmill exercise increased motor and memory functions in the LPS-induced CP rats.

Neurogenesis in the hippocampus plays a critical role in memory process. It is well known that treadmill exercise increased 

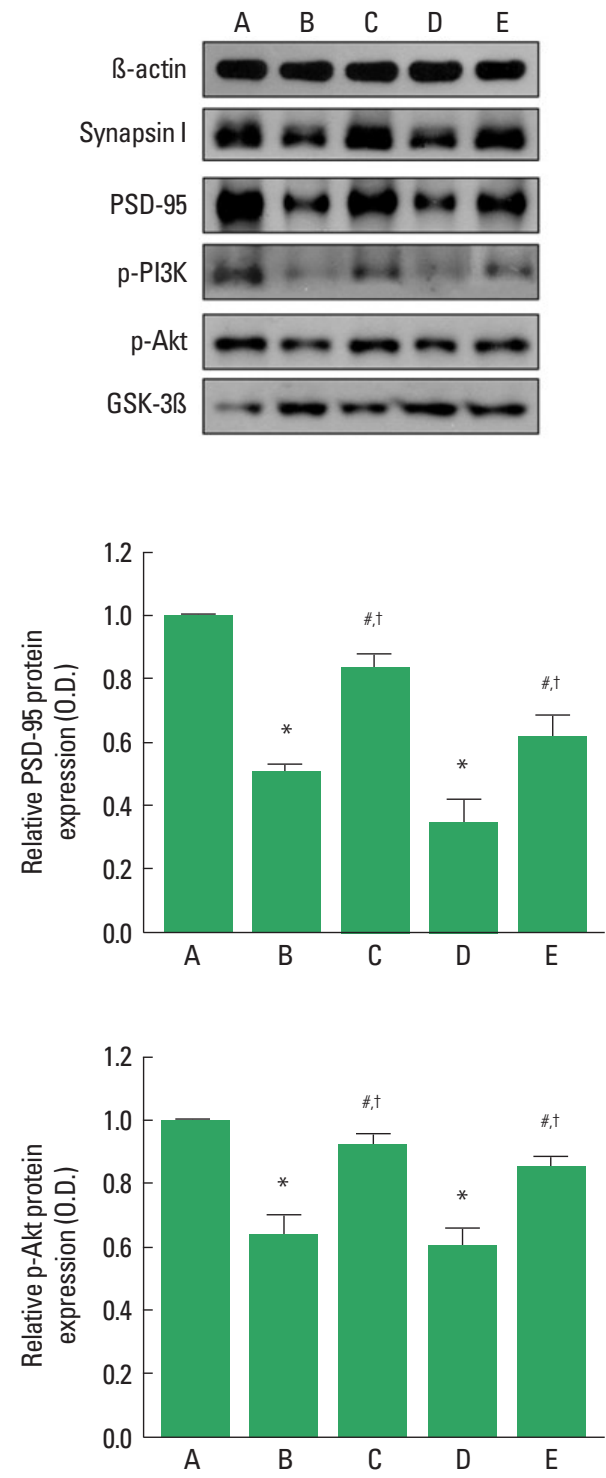
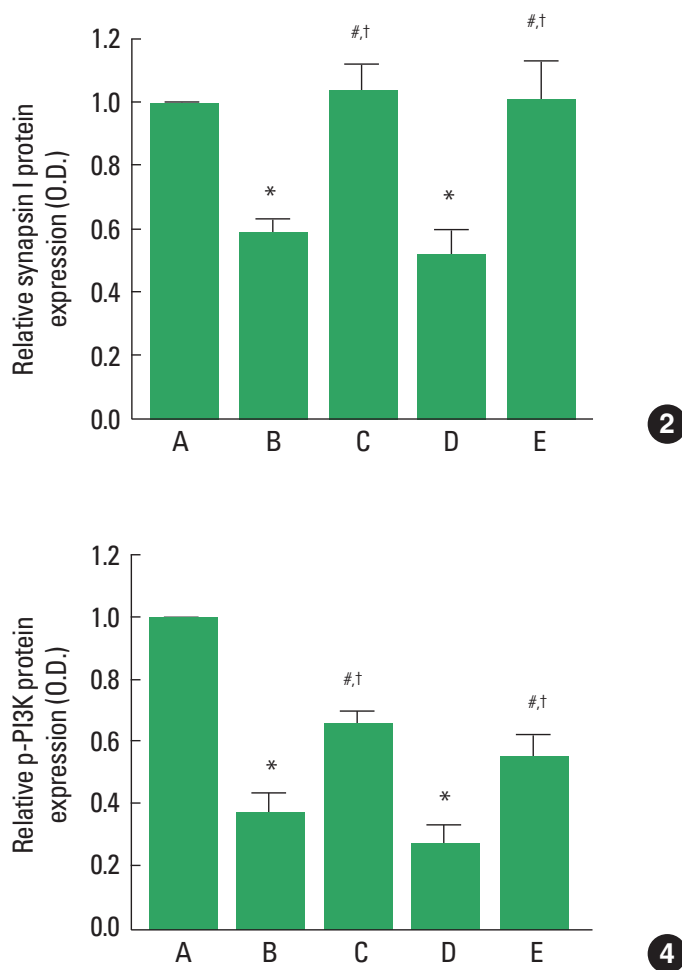

$(4)$

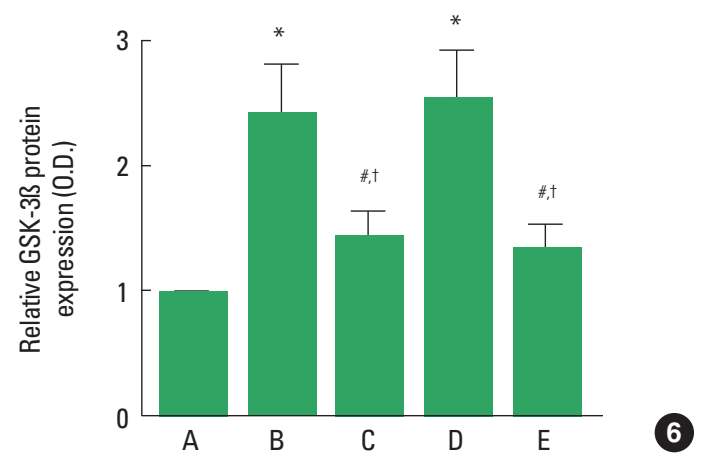

Fig. 5. Effects of treadmill exercise on synapsin I, postsynaptic density-95 (PSD-95), phosphorylated-phosphoinositide 3 kinase (p-PI3K), phosphorylated-Akt (p-Akt),

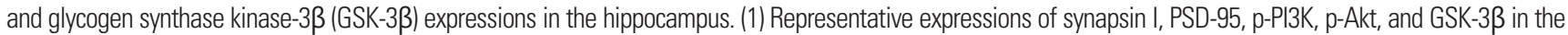
hippocampus. (2-6) Relative expressions of synapsin I, PSD-95, PI3K, Akt, and GSK-33 ratio in the hippocampus. A, control group; B, cerebral palsy group; C, cerebral palsy and exercise group; $\mathrm{D}$, cerebral palsy and sensorimotor restriction group; $\mathrm{E}$, cerebral palsy, sensorimotor restriction, and exercise group. The data are presented as the mean \pm standard error of the mean. ${ }^{*} P<0.05$ compared to the control group. ${ }^{\sharp} P<0.05$ compared to the cerebral palsy group. ${ }^{\dagger} P<0.05$ compared to the cerebral palsy and sensorimotor restriction group.

neurogenesis in the hippocampus under the normal and various pathological conditions (Shin et al., 2013; van Praag et al., 2005). In the present study, treadmill exercise increased hippocampal cell proliferation in the LPS-induced CP rats.

Physical exercise increased synaptic plasticity markers in the hippocampus, such as synapsin I and PSD-95 (Vaynman et al., 2003). Synapsin I is a presynaptic phosphoprotein and regulates neurotransmitter release and axonal elongation (Jovanovic et al.,
2000). And PSD-95 contributes maturation of excitatory synapses (El-Husseini et al., 2000). In the present study, treadmill exercise enhanced the expressions of synapsin I and PSD-95 in the LPS-induced $\mathrm{CP}$ rats.

The PI3k-Akt pathway is associated with a various functions, such as glucose metabolism, protein synthesis, and cell proliferation (Brazil et al., 2004). Chen and Russo-Neustadt, (2005) suggested that physical exercise activated the PI3k-Akt pathway in 
the hippocampus, that is implicated in the enhancing neuronal survival. In the present study, treadmill exercise enhanced the expressions of $\mathrm{p}-\mathrm{PI} 3 \mathrm{~K}$ and $\mathrm{p}-\mathrm{Akt}$ in the LPS-induced $\mathrm{CP}$ rats.

Physiological exercise activates PI3k-Akt pathway, and GSK-3 phosphorylation was inhibited (Sakamoto et al., 2003). GSK-3 $\beta$ negatively regulates proliferation of neural precursors and neurogenesis (Nedachi et al., 2011). In the present study, treadmill exercise suppressed GSK-3 $\beta$ expression in the LPS-induced CP rats.

PI3K-Akt pathway in the brain is implicated in synaptic plasticity, cognitive function, and neurogenesis (Gasparini et al., 2002). The present results suggest that treadmill exercise might improve motor and memory functions in the $\mathrm{CP}$ patients. These effects of treadmill exercise might be ascribed to the activation of PI3K-Akt pathway by treadmill running, thereby increasing neurogenesis through enhancing synaptic plasticity.

\section{CONFLICT OF INTEREST}

No potential conflict of interest relevant to this article was reported.

\section{ACKNOWLEDGMENTS}

This work was supported by the National Research Foundation of Korea Grant funded by the Korean Government (NRF-2014S1 A5A2A01016136).

\section{REFERENCES}

Bax M, Goldstein M, Rosenbaum P, Leviton A, Paneth N, Dan B, Jacobsson B, Damiano D; Executive Committee for the Definition of Cerebral Palsy. Proposed definition and classification of cerebral palsy, April 2005. Dev Med Child Neurol 2005;47:571-576.

Brazil DP, Yang ZZ, Hemmings BA. Advances in protein kinase B signalling: AKTion on multiple fronts. Trends Biochem Sci 2004;29:233-242.

Chen MJ, Russo-Neustadt AA. Exercise activates the phosphatidylinositol 3-kinase pathway. Brain Res Mol Brain Res 2005;135:181-193.

Coq JO, Strata F, Russier M, Safadi FF, Merzenich MM, Byl NN, Barbe MF. Impact of neonatal asphyxia and hind limb immobilization on musculoskeletal tissues and S1 map organization: implications for cerebral palsy. Exp Neurol 2008;210:95-108.

Damiano DL. Activity, activity, activity: rethinking our physical therapy approach to cerebral palsy. Phys Ther 2006;86:1534-1540.

Damiano DL, Alter KE, Chambers H. New clinical and research trends in lower extremity management for ambulatory children with cerebral palsy. Phys Med Rehabil Clin N Am 2009;20:469-491.

Derrick M, Luo NL, Bregman JC, Jilling T, Ji X, Fisher K, Gladson CL, Beardsley DJ, Murdoch G, Back SA, Tan S. Preterm fetal hypoxia-ischemia causes hypertonia and motor deficits in the neonatal rabbit: a model for human cerebral palsy? J Neurosci 2004;24:24-34.

El-Husseini AE, Schnell E, Chetkovich DM, Nicoll RA, Bredt DS. PSD-95 involvement in maturation of excitatory synapses. Science 2000;290: 1364-1368.

Gasparini L, Netzer WJ, Greengard P, Xu H. Does insulin dysfunction play a role in Alzheimer's disease? Trends Pharmacol Sci 2002;23:288293.

Gilstrap LC 3rd, Ramin SM. Infection and cerebral palsy. Semin Perinatol 2000;24:200-203.

Jovanovic JN, Czernik AJ, Fienberg AA, Greengard P, Sihra TS. Synapsins as mediators of BDNF-enhanced neurotransmitter release. Nat Neurosci 2000;3:323-329.

Jung SY, Kim DY, Yune TY, Shin DH, Baek SB, Kim CJ. Treadmill exercise reduces spinal cord injury-induced apoptosis by activating the PI3K/ Akt pathway in rats. Exp Ther Med 2014;7:587-593.

Kim DY, Jung SY, Kim TW, Lee KS, Kim K. Treadmill exercise decreases incidence of Alzheimer's disease by suppressing glycogen synthase kinase-3 $\beta$ expression in streptozotocin-induced diabetic rats. J Exerc Rehabil 2015;11:87-94.

Marcuzzo S, Dutra MF, Stigger F, do Nascimento PS, Ilha J, Kalil-Gaspar PI, Achaval M. Different effects of anoxia and hind-limb immobilization on sensorimotor development and cell numbers in the somatosensory cortex in rats. Brain Dev 2010;32:323-331.

Marques MR, Stigger F, Segabinazi E, Augustin OA, Barbosa S, Piazza FV, Achaval M, Marcuzzo S. Beneficial effects of early environmental enrichment on motor development and spinal cord plasticity in a rat model of cerebral palsy. Behav Brain Res 2014;263:149-157.

Nedachi T, Kawai T, Matsuwaki T, Yamanouchi K, Nishihara M. Progranulin enhances neural progenitor cell proliferation through glycogen synthase kinase $3 \beta$ phosphorylation. Neuroscience 2011;185:106-115.

Sakamoto K, Aschenbach WG, Hirshman MF, Goodyear LJ. Akt signaling in skeletal muscle: regulation by exercise and passive stretch. Am J Physiol Endocrinol Metab 2003;285:E1081-1088.

Shin MS, Ko IG, Kim SE, Kim BK, Kim TS, Lee SH, Hwang DS, Kim CJ, Park JK, Lim BV. Treadmill exercise ameliorates symptoms of methimazole-induced hypothyroidism through enhancing neurogenesis and suppressing apoptosis in the hippocampus of rat pups. Int J Dev Neurosci 2013;31:214-223.

Stigger F, Felizzola AL, Kronbauer GA, Couto GK, Achaval M, Marcuzzo S. Effects of fetal exposure to lipopolysaccharide, perinatal anoxia and sensorimotor restriction on motor skills and musculoskeletal tissue: 
implications for an animal model of cerebral palsy. Exp Neurol 2011; 228:183-191.

Strata F, Coq JO, Byl N, Merzenich MM. Effects of sensorimotor restriction and anoxia on gait and motor cortex organization: implications for a rodent model of cerebral palsy. Neuroscience 2004;129:141-156.

Tiwari SK, Seth B, Agarwal S, Yadav A, Karmakar M, Gupta SK, Choubey V, Sharma A, Chaturvedi RK. Ethosuximide Induces Hippocampal Neurogenesis and Reverses Cognitive Deficits in an Amyloid- $\beta$ Toxin-induced Alzheimer Rat Model via the Phosphatidylinositol 3-Kinase (PI3K)/Akt/Wnt// 3 -Catenin Pathway. J Biol Chem 2015;290:2854028558.

van Praag H, Shubert T, Zhao C, Gage FH. Exercise enhances learning and hippocampal neurogenesis in aged mice. J Neurosci 2005;25:86808685.
Vaynman S, Ying Z, Gomez-Pinilla F. Interplay between brain-derived neurotrophic factor and signal transduction modulators in the regulation of the effects of exercise on synaptic-plasticity. Neuroscience 2003;122:647-657.

Wu Y, Peng H, Cui M, Whitney NP, Huang Y, Zheng JC. CXCL12 increases human neural progenitor cell proliferation through Akt-1/ FOXO3a signaling pathway. J Neurochem 2009;109:1157-1167.

Ying Z, Roy RR, Zhong H, Zdunowski S, Edgerton VR, Gomez-Pinilla F. BDNF-exercise interactions in the recovery of symmetrical stepping after a cervical hemisection in rats. Neuroscience 2008;155:1070-1078.

Zhang L, Hu X, Luo J, Li L, Chen X, Huang R, Pei Z. Physical exercise improves functional recovery through mitigation of autophagy, attenuation of apoptosis and enhancement of neurogenesis after MCAO in rats. BMC Neurosci 2013;14:46. 